\title{
New scoring system for melanoma accurately stratifies risk
}

In melanoma, biospy of the lymph nodes directly draining the primary site (sentinel lymph nodes; SNs) is used to select patients at risk of metastatic disease in non-sentinel nodes (NSNs). This assessment is then used to decide whether an individual should undergo complete lymph-node dissection (CLND). However, only $20 \%$ of the patients that undergo CLND have metastatic disease. A study aiming to improve the accuracy of this risk assessment has produced a novel scoring system for these patients.

Lead author of the study, Rajmohan Murali, explained: "accurate prediction of the likelihood of NSN involvement would be helpful for determining prognosis, for planning management, and potentially for identifying patients in whom CLND can be safely avoided because the risk of NSN positivity is very low." The study assessed 409 patients with melanoma with confirmed positive SNs. The primary tumor characteristics and morphological features were assessed and a score was defined. The score is described by Murali: "the final N-SNORE incorporates clinical (sex), primary tumor (regression), $\mathrm{SN}$ (proportion of involved SNs), and histological features of SN deposits ... and is a novel means of accurately stratifying risk of NSN positivity in SN-positive melanomas."

The N-SNORE scoring system will need to be validated in other patient cohorts before it can be recommended for use. However, if validated, it is possible that it may be used to identify high-risk patients and those that can safely be spared CLND.

Rebecca Kirk

Original article Murali, R. et al. Non-sentinel node risk score (N-SNORE): a scoring system for accurately stratifying risk of non-sentinel node positivity in patients with cutaneous melanoma with positive sentinel lymph nodes. J. Clin. Oncol. 28, 4441-4449 (2010) 